\title{
Behavioral Profiles of Affected and Unaffected Siblings of Children with Autism: Contribution of Measures of Mother-Infant Interaction and Nonverbal Communication
}

\author{
Agata Rozga - Ted Hutman • Gregory S. Young • \\ Sally J. Rogers · Sally Ozonoff • Mirella Dapretto • \\ Marian Sigman
}

Published online: 22 June 2010

(C) The Author(s) 2010. This article is published with open access at Springerlink.com

\begin{abstract}
We investigated whether deficits in social gaze and affect and in joint attention behaviors are evident within the first year of life among siblings of children with autism who go on to be diagnosed with autism or ASD (ASD) and siblings who are non-diagnosed (NoASD-sib) compared to low-risk controls. The ASD group did not differ from the other two groups at 6 months of age in the frequency of gaze, smiles, and vocalizations directed toward the caregiver, nor in their sensitivity to her withdrawal from interaction. However, by 12 months, infants in the ASD group exhibited lower rates of joint attention and requesting behaviors. In contrast, NoASD-sibs did not differ from comparison infants on any variables of interest at 6 and 12 months.
\end{abstract}

Keywords Autism · Broader autism phenotype . Early identification · Mother-infant interaction . Still face procedure $\cdot$ Nonverbal communication

\footnotetext{
A. Rozga - T. Hutman · M. Dapretto · M. Sigman Semel Institute for Neuroscience and Human Behavior, University of California, Los Angeles, CA, USA

G. S. Young $\cdot$ S. J. Rogers $\cdot$ S. Ozonoff

M.I.N.D. Institute, Department of Psychiatry and Behavioral Sciences, School of Medicine, University of California, Davis, CA, USA

A. Rozga $(\bowtie)$

School of Interactive Computing, Georgia Institute of Technology, 85 5th Street NW, Atlanta, GA 30332, USA e-mail: agata@gatech.edu
}

\section{Introduction}

Siblings of children with autism are at heightened risk for developing an autism spectrum disorder (ASD) (Fombonne 2003; Ritvo et al. 1989) and milder difficulties in the domains of social interaction and communication (Bailey et al. 1998; Bolton et al. 1994; Pickles et al. 2000). Prospective study of the development of these infants from the first months of life has thus increasingly been used as a controlled and efficient method for investigating both early signs of autism (Landa et al. 2007; Sullivan et al. 2007; Zwaigenbaum et al. 2005) and the broader autism phenotype (Cassel et al. 2007; Toth et al. 2007; Yirmiya et al. 2006). In the present longitudinal study, we explored whether measures of social-communicative behaviors exhibited while interacting with a caregiver at 6 months of age, and joint attention and requesting behaviors in an object-mediated interaction with an examiner at 12 months, tap early manifestations of autism and the broader autism phenotype in this group.

Face-to-face interactions between infants and their caregivers provide a natural and well-characterized context within which to examine infants' earliest capacities for engaging with others. By 6 months of age, typically developing infants use combinations of expressive behavior in multiple modalities, including vocalization, facial expression, and visual orientation (Messinger et al. 1999; Weinberg and Tronick 1994; Yale et al. 2003) and coordinate the timing of these behaviors with those of their interactive partners (Cohn and Tronick 1988; Feldman 2003; Jaffe et al. 2001). In contrast, research with preschool age and older children with autism suggests they rarely integrate gaze, gestures and affective displays to communicate social intention (Dawson et al. 1990; Joseph and Tager-Flusberg 1997; Kasari et al. 1990; Trad et al. 
1993). Studying face-to-face interactions between infants at risk for autism and their caregivers provides a unique opportunity to examine whether deficits in the social use of gaze and affect may be apparent as early as 6 months of age.

To date, three groups have examined infants' socialcommunicative behaviors in the context of interacting with the caregiver among infant siblings of children with autism (Cassel et al. 2007; Ibanez et al. 2008; Merin et al. 2007; Yirmiya et al. 2006). In all four studies, infants participated in the Still Face Procedure (SFP), a structured paradigm in which the caregiver is asked to withdraw completely from interacting with the infant and assume a neutral expression for a pre-specified period of time before resuming contingent interaction (Tronick et al. 1978). Infants typically react to the caregiver's still face with significant decrease in eye contact and smiling and an increase in negative affect (Adamson and Frick 2003), a reaction that has been interpreted as reflecting their appreciation of a disruption of positive co-regulation and a violation of social expectations (Hains and Muir 1996; Tronick and Cohn 1989). Employing the traditional face-to-face SFP, researchers found that compared to low-risk controls, 6-month-old siblings of children with autism smiled significantly less prior the caregiver's withdrawal from interaction, displayed significantly more neutral affect when their caregiver resumed interaction (Cassel et al. 2007), and produced fewer gaze shifts to and away from the caregiver across the SFP (Ibanez et al. 2008). At 4 months, these infants displayed significantly more neutral affect across all three episodes of the SFP (Yirmiya et al. 2006). Merin et al. (2007) used eye tracking to examine infants' gaze patterns during the SFP as they interacted with caregivers via a closed-circuit TV-video system. They identified a subgroup of infants who spent a greater amount of time looking at their mother's mouth relative to her eyes, with all but one infant in this subgroup coming from the autism sibling group.

Although some differences in gaze and affective expression between autism siblings and low-risk controls have been reported across these studies, these differences were not tied to the still face episode, suggesting that both groups of infants perceived and reacted to a break in contingent interaction with the caregiver. However, as only one infant in the Yirmiya study was subsequently diagnosed with autism and diagnostic outcome data was not available for infants in the Cassel and Ibanez samples, no definitive conclusions can be drawn regarding the extent to which early social communicative behaviors may be disrupted in infants who go on to receive a diagnosis of autism. Indeed, in a follow-up of the Merin et al. sample, none of the infants in the high-risk group who were subsequently diagnosed with autism were in the subgroup that looked mostly to the mouth at 6 months of age (Young et al. 2009). These results suggest that atypical behaviors identified among infant siblings of children with autism as $a$ group may not be informative regarding early signs of autism. Consequently, we examined whether measures of infants' gaze and affect during the SFP and during a free play interaction with the caregiver at 6 months of age capture early social impairment among autism siblings who are subsequently diagnosed with an ASD compared to siblings whose outcomes reflect typical development and to low-risk controls.

While a mutual sharing of attention and affect during face-to-face social exchanges characterizes the earliest social interactions, toward the end of the first year of life infants begin to coordinate their attention and interest with others about external objects and events (Bakeman and Adamson 1984; Bruner and Sherwood 1983; Carpenter et al. 1998). Such joint attention behaviors represent a core area of impairment for children with autism well into the preschool years and beyond. Compared to typically developing or developmentally delayed children, children with autism are less likely to use eye contact, affect, and gestures to share and direct their interactive partners' attention to objects of interest, as well as to respond to the attention-directing cues of others. Their use of these behaviors for more instrumental purpose, such as pointing to obtain an object, is less impaired (Dawson et al. 2004; Mundy et al. 1986; Sigman and Ruskin 1999).

A growing body of research suggests that these deficits may be among the earliest diagnostic indicators of ASD (Baranek 1999; Charman et al. 1997; Osterling and Dawson 1994). Longitudinal studies of infant siblings of children with autism indicate that measures of joint attention and requesting skill may be sensitive to early manifestations of autism and the broader phenotype from 14 to 24 months (Cassel et al. 2007; Landa et al. 2007; Mitchell et al. 2006; Sullivan et al. 2007; Toth et al. 2007; Yirmiya et al. 2006). However, the particular domains affected and the ages at which group differences emerge vary greatly across studies, particularly with respect to those siblings who do not go on to be diagnosed with autism. The present study focuses on a large sample of both affected and unaffected siblings of children with autism with the aim of clarifying the extent to which specific deficits in joint attention, or more general deficits in nonverbal communication that include both sharing/directing attention and requesting behaviors, may be observed in these two groups at 12 months of age.

We hypothesized that infants who go on to be diagnosed with autism or ASD will direct fewer looks, smiles, and vocalizations toward their caregiver at 6 months of age than low-risk controls, and that they will be less affected by the disruption of contingent interaction in the SFP, as 
manifested through less gaze aversion and negative affect in the "still face" portion of the procedure. We further hypothesized that at 12 months, these infants will exhibit deficits in initiating joint attention and responding to distal points. Broader autism phenotype effects, reflected in comparisons of non-affected autism siblings and low-risk controls, were expected to manifest in more subtle, qualitatively similar differences, including a diminished still face effect and less pronounced nonverbal communication deficits.

\section{Methods}

\section{Participants}

Participants were recruited through an ongoing longitudinal study of the development of infant siblings of children with autism (high-risk group) or typical development (lowrisk group), conducted at UCLA and UC Davis. Infants entered the study at $6,{ }^{1} 12$, or 18 months of age and, depending on age of entry, were re-assessed at 12, 18, and 24 , and 36 months. The sub-sample described in the current report $(n=167)$ includes infants recruited by 6 or 12 months of age who had diagnostic outcome data available for at least one time-point between 24 and 36 months.

Eligibility criteria included English as the primary language spoken in the home, and birth at or after 36 weeks of gestation. Further inclusion criteria for the high-risk group included at least one older sibling with a previous clinical diagnosis on the autism spectrum, with no other medical conditions associated with autism (e.g., Fragile X, Tuberous Sclerosis). Diagnoses of ASD in the older siblings were confirmed using the Autism Diagnostic Interview-Revised (ADI-R; Lord et al. 1994), the Autism Diagnostic Observation Schedule (ADOS; Lord et al. 2000), and DSM-IV criteria (APA 1994) at UCLA, and via the ADOS and Social Communication Questionnaire (SCQ; Rutter et al. 2003) at UC Davis. Inclusion criteria for the low-risk group included lack of developmental, learning, or medical conditions in any older sibling, and no ASD diagnoses in the extended family. Parents of low-risk infants additionally completed the SCQ to rule out autistic symptomatology in all older siblings.

Descriptive information about the sample is provided in Table 1. The composition of the samples of infants varies across the three sets of analyses reported herein, with much overlap between the samples. Details regarding sample

\footnotetext{
${ }^{1}$ A subset of the infants in the low-risk control group $(n=45)$ and the autism sibling group $(n=11)$ were recruited prior to 6 months of age.
}

sizes across analyses and missing data are provided in Table 2 .

\section{Developmental Assessments}

The Mullen Scales of Early Learning (MSEL; Mullen 1995) was administered at each visit. The MSEL is a normed standardized developmental measure of language and cognitive functioning that provides age equivalent and standard scores $(M=50, S D=10)$ from birth to 68 months of age on four separate subscales: visual reception, fine motor, expressive language, and receptive language. It also provides an overall standardized score of intellectual functioning, the Early Learning Composite $(M=100, S D=15)$. Age equivalents from the two language domain scores were averaged to generate a verbal mental age, and age equivalents from the fine motor and visual reception domain scores were averaged to generate nonverbal mental age (see Table 1).

\section{Diagnostic Classification}

Infants were screened for autistic symptomatology at 18 , 24, and 36 months using the ADOS (Lord et al. 2000), a structured observational measure comprised of play-based activities that elicit behaviors relevant to a diagnosis of autism spectrum disorders. Diagnostic classification on the ADOS is generated by means of an empirically derived algorithm, using score thresholds in social reciprocity, communication, as well as a combination of these two domains. Diagnostic algorithm cut-off scores are provided both for Autism and Autism Spectrum. Examiners were trained and confirmed reliable to administer and score the ADOS according to its authors' specifications.

In addition, at each site a clinician reviewed the case files of all participants who demonstrated features of ASD at any time point. Based on the child's ADOS scores and behavioral observations of the child's behavior during testing, the clinician determined whether the child met diagnostic criteria for autism or autism spectrum disorder utilizing symptom criteria outlined in the DSM-IV.

For the purposes of the present analyses, children's diagnostic outcome classifications were based on their ADOS scores and clinician judgment at 36 months $(n=148)$ or, in cases where an infant had not been seen for the 36-month assessment visit $(n=19)$, their 24-month ADOS scores and DSM-IV criteria. Three groups were generated. The ASD group consists of 17 infants, all of whom met criteria for Autism or Autism Spectrum at 36 months based on ADOS and clinician judgment. 15 of these infants are in the high-risk group, and the remaining two initially enrolled in the low-risk group. The NoASDsib group consists of 84 infants in the high-risk group who 
Table 1 Sample characteristics

\begin{tabular}{|c|c|c|c|}
\hline & ASD & NoASD-sib & $\mathrm{TD}$ \\
\hline Gender ( $\%$ male $)$ & 82 & 44 & 55 \\
\hline Race (\% white) & 65 & 62 & 64 \\
\hline \multicolumn{4}{|l|}{ Maternal education $^{\mathrm{a}}$} \\
\hline High school/some college (\%) & 25 & 22 & 15 \\
\hline College degree $(\%)$ & 62 & 40 & 40 \\
\hline Graduate/professional degree $(\%)$ & 13 & 38 & 45 \\
\hline \multicolumn{4}{|l|}{ Family income ${ }^{\mathrm{b}}$} \\
\hline Less than $\$ 50 \mathrm{k}(\%)$ & 25 & 14 & 19 \\
\hline$\$ 50 \mathrm{k}$ to $\$ 100 \mathrm{k}(\%)$ & 31 & 39 & 25 \\
\hline More than $\$ 100 \mathrm{k}(\%)$ & 44 & 47 & 56 \\
\hline \multicolumn{4}{|l|}{ 6-month analyses } \\
\hline Chronological age & $6.3(0.9)$ & $6.1(0.5)$ & $6.2(0.2)$ \\
\hline Verbal mental age & $5.9(1.5)$ & $5.6(0.7)$ & $5.8(0.8)$ \\
\hline Nonverbal mental age & $6.8(2.3)$ & $6.8(1.2)$ & $7.0(1.2)$ \\
\hline \multicolumn{4}{|l|}{ 12-month analyses } \\
\hline Chronological age & $12.0(0.4)$ & $12.2(0.4)$ & $12.1(0.3)$ \\
\hline Verbal mental age & $9.8(1.7)^{* *}$ & $12.0(1.8)$ & $11.8(2.0)$ \\
\hline Nonverbal mental age & $13.4(1.3)^{* *}$ & $14.8(1.5)$ & $14.8(1.5)$ \\
\hline \multicolumn{4}{|l|}{ Outcome visit ${ }^{\mathrm{c}}$} \\
\hline ADOS communication + social total & $13.7(3.3)^{* *}$ & $2.9(2.2)^{*}$ & $2.1(2.1)$ \\
\hline Expressive language standard score & $42.6(14.2)^{* *}$ & $53.6(9.0)^{*}$ & $56.7(8.1)$ \\
\hline Receptive language standard score & $40.5(14.1)^{* *}$ & $51.1(11.3)$ & $53.0(11.0)$ \\
\hline Early Learning Composite & $84.3(24.7)^{* *}$ & $109.5(17.6)$ & $108.4(19.7)$ \\
\hline
\end{tabular}

Table 2 Sample sizes for enrollment, analysis, missing data, and age at outcome

\begin{tabular}{lrrr}
\hline & ASD & NoASD-sib & TD \\
\hline Sample & & & \\
Enrolled at $\leq 6$ months & 15 & 62 & 61 \\
Enrolled at 12 months & 2 & 22 & 5 \\
Total & 17 & 84 & 66 \\
6-month analyses & & & \\
Data available & & 46 & 38 \\
Missing SF & 9 & 0 & 10 \\
Missing MCX & 0 & 5 & 3 \\
12-month analyses & 1 & & \\
Data available & 17 & 84 & 66 \\
Missing ESCS & 0 & 3 & 59 \\
Outcome visit & & & 7 \\
36 months & 17 & 73 & \\
24 months & 0 & 11 & \\
\hline
\end{tabular}

$S F$ still face procedure, $M C X$ mother-infant free play interaction, ESCS early social communication scales

${ }^{a}$ The discrepancy between the number of infants enrolled at 6 months and the number available for 6-month analyses stems from the fact 45 infants (6 in the ASD group, 16 in the NoASD-sib group, and 23 in the TD group) enrolled in the study at 6 months, but this occurred after the 6-month coding had already been completed did not meet criteria for Autism or ASD based on the ADOS and DSM-IV criteria. All but 12 of the infants were seen through 36 months. The TD group consists of 66 infants in the low-risk group, none of whom met criteria for autism or ASD based on the ADOS and clinician judgment. All but 7 of the infants were seen through 36 months.

Infants in the high-risk group whose outcome diagnosis was based on the 24-month visit were included in the NoASD-sib group on the basis of their 24-month ADOS scores and a clinician's judgment that there were no concerns of ASD. Two factors further justify the inclusion of these infants in the NoASD-sib outcome group. Of the infants in the NoASD-sib group whose outcomes were confirmed at 36 months, none raised concerns of ASD when evaluated by a clinician at the 24-month visit. In contrast, all of the infants in the ASD group were flagged by clinician judgment as showing signs of ASD at 24 months. Based on these data, high-risk infants who did not meet ADOS cutoffs and did not raise clinical concerns at 24 months were included in the non-affected autism sibling group.

The ADOS communication and social interaction total score and measures of developmental functioning from the MSEL at outcome for the three groups of infants are provided in Table 1. 


\section{Free Play Mother-Infant Interaction at 6 Months}

Infants were seated in an infant seat placed on a table, and mothers were asked to sit in a chair, facing their infant. Mothers were instructed to interact with the infant for 1 min without touching the infant and without using any toys. The purpose of this interaction was to examine the extent to which infants would direct attention and affect toward their caregiver without the additional aid of objects and physical stimulation. The interactions were videotaped with a split screen, with one camera focused on the infant and a second camera focused on the caregiver. Motherinfant interaction data were not available for one infant in the ASD group, five infants in the NoASD-sib group, and three infants in the TD group due to infant fussiness, deviations from the protocol, and video capture issues.

Infant behaviors were subsequently coded during separate passes through the video by coders who remained blind to infants' group affiliation and developmental outcomes. Onsets and offsets of the following behaviors were recorded: gaze to mother's face, smile, and non-distress vocalization. Inter-rater reliability, based on the percentage of overlap in the duration of each behavior in real time (from onset to offset of each behavior), was $95 \%$ for infant gaze, $85 \%$ for infant smile, and $93 \%$ for infant vocalization. Kappa coefficients for agreement on onset and offset times within each behavior category, with a tolerance window of $1 \mathrm{~s}$, were as follows: .90 for gaze, .82 for smile, and .83 for vocalization.

Based on the individual behaviors, a number of variables were derived. These included frequencies of each of the infant behaviors noted above (i.e., the number of onsets for each behavior, regardless of its duration) and the total duration of each behavior. Two additional behaviors were defined based on combinations of individual behaviors: social smile (smile co-occurring with gaze to the mother's face) and social vocalization (non-distress vocalization co-occurring with gaze to the mother's face). Because the length of the interaction varied across dyads $(M=62.4 \mathrm{~s}$, $S D=11.5$ ), frequencies and durations of all behaviors were converted, respectively, to rates per minute and percent durations.

\section{Still Face Procedure at 6 Months}

Following the free-play interaction, infants and their caregivers participated in a modified version of Tronick's (Tronick et al. 1978) Still Face Procedure (SFP). Briefly, this procedure consisted of another 1-min free play episode (parents were allowed to touch the infant), followed by a 1-min still face episode during which the mother was asked to withdraw from the interaction, assume a neutral expression, and to remain unresponsive, and ended with a 1-min reunion episode during which the mother was instructed to re-engage her baby. Infants remained in the infant seat for the duration of the SFP. The steps of the SFP procedure were thoroughly explained to the caregiver prior to the beginning of the interaction. An examiner sat behind the infant and in view of the caregiver in order to unobtrusively signal when the caregiver was to move from one SFP episode to the next. Mother and infant were videotaped in the manner noted above for the free play interaction.

Infants' gaze and affect in the SFP were coded in two separate passes through the video by a coder blind to infants' group affiliation and outcome. SFP data were not coded for ten infants in the TD group due to infant fussiness, deviations from the protocol, and video capture issues. Based on the coding system employed in the Yirmiya et al. (2006) study, gaze behavior was coded in 1-s intervals into one of five mutually exclusive and exhaustive categories: gaze to the mother's face, gaze to the mother's body (not including face), gaze to object (e.g., infant seat strap), averted gaze (away from the mother's face and body and not focused on an identifiable object), and eyes closed. Kappa coefficients indicated good reliability for infant gaze behaviors for the three episodes: .88 for the social play episode, .90 for the still face episode, and .92 for the reunion episode. Infant facial affect was coded in 5-s intervals into one of six mutually exclusive and exhaustive categories: very negative, negative, neutral/interest, positive, and very positive. Due to the very low frequency of very positive and very negative affect, the six affect categories were reduced as follows: negative affect (very negative and negative), neutral (neutral/interest), and positive (very positive and positive). Inter-rater reliability for affect, as assessed by Kappa coefficients, was .83 for the free play episode, .85 for the still face episode, and .83 for the reunion episode.

\section{Nonverbal Communication at 12 Months}

Infants were administered the Early Social Communication Scales (ESCS; Mundy et al. 2003), a 15-25 min structured observation that measures triadic nonverbal communication skills. ${ }^{2}$ The child and an examiner sat facing each other at a small table, with a set of toys (e.g., wind-up, trapeze, balloon) in view but out of the child's reach. The

\footnotetext{
${ }^{2}$ In line with previous literature, we use the term nonverbal communication to refer to triadic nonverbal communication skills (i.e., between two people in reference to objects or events) measured by the ESCS. We draw a conceptual distinction from the face-to-face nonverbal communication that is the focus of the 6-month analyses.
} 
examiner presented the toys to the child one at a time and played a series of games aimed at eliciting turn taking. At two points during the procedure, the examiner pointed to posters to the left, right, and behind the child while saying the child's name emphatically three times. Finally, the examiner brought out a picture book and pointed to three pictures on 3 successive pages while saying the child's name.

Trained observers recorded frequencies of child communicative acts falling into one of three mutually exclusive categories, derived from a coding system developed by Mundy et al. (2003). Initiating Joint Attention (IJA) refers to the child's use of gaze and gesture to initiate shared attention with the examiner about objects or events of interest to the child. IJA behaviors were grouped into lowlevel joint attention (IJA_low), which included making eye contact with the examiner while manipulating a toy and alternating gaze between an active toy and the examiner, and high-level joint attention (IJA_high), which included pointing to objects of interest (with or without eye contact) or clearly holding up an object to show it to the examiner. Initiating Behavior Regulation (IBR) refers to the child's use of eye contact and gesture to elicit aid in obtaining or activating a toy. Low-level requesting behaviors (IBR_low) included making eye contact to request a toy, or reaching or otherwise motioning toward a desired toy (with or without eye contact). High-level requesting behaviors (IBR_high) included pointing to a desired, out-of-reach toy or giving an object to the examiner for the purpose of obtaining assistance with operating it (with or without eye contact). Responding to Joint Attention (RJA) refers to the child's ability to follow the tester's line of regard and pointing gestures, and consisted of the number of times the child turned his gaze in the direction of the examiner's points to posters on the wall (RJA_dist; $\max =6$ ) and pictures in a book (RJA_prox; $\max =9$ ). Reliability was assessed via intra-class correlations (Shrout and Fleiss 1979) based on $15 \%$ of the sample; these ranged from .91 to .97 , with a mean of .94 .

ESCS data were not available for three infants in the NoASD-sib group and six infants in the TD group due to video capture issues and, in one case, a missed appointment. Due to varying degrees of infants' interest in the toys and differences in the amount of time each toy was presented to the child, the length of the ESCS varied between 9.2 and $34.8 \min (M=18.9, S D=4.9)$. There were group differences in the length of the ESCS. ${ }^{3}$ Thus, the four IJA

\footnotetext{
3 The average length of the ESCS for infants in the ASD group $(M=21.2, S D=4.9)$ was significantly longer than the NoASD-sibs $(M=17.9, S D=3.7)$ but it did not differ from the TD controls $(M=19.4, S D=5.6)$. The effect size for this difference is small $\left(\eta_{\mathrm{p}}^{2}=.05\right)$.
}

and IBR behaviors were expressed as rates per minute with respect to the total duration of the ESCS. A subset of participants was administered fewer than the required number of point-following trials due to changes in the study protocol (2 ASD, 11 NoASD-sib, 1 TD) and experimenter error (1 ASD, 5 NoASD-sib, 2 TD). Nevertheless, the number of correct responses to the point-following tasks was not correlated with the total number of trials administered, both in the sample as a whole $(r=-.02$, $p=.78)$ and in the sub-sample of infants with fewer than the required number of trials $(r=-.23, p=.28)$. Moreover, there was no difference between the groups in the proportion of infants with fewer than 6 trials, $\chi^{2}$ $(n=2)=2.2, p=.33$.

\section{Data Analytic Plan}

Variables from the free-play interaction, SFP, and ESCS represent frequencies of individual infant behaviors, and were modeled as count data using the Poisson Regression Model (PRM) or, in cases of significant overdispersion (i.e., variance $>$ mean), the Negative Binomial Regression Model (NBRM). The interpretation of these models involves assessing how changes in the independent variables affect the conditional mean and the probabilities of various counts on the dependent variable. The appropriateness of using count models over standard linear models to analyze the data was confirmed through visual inspection of the distribution of these variables and skewness statistics, which indicated most variables were non-normally distributed with significant positive skew, thus violating the distributional assumptions of ordinary least squares regression (Long and Freese 2006). A large number of zero counts for many variables precluded data transformations to satisfy the distributional assumptions of ANOVA.

Data from the SFP were analyzed with random effects NBRM, with episode as the repeated measure, utilizing the xtnbreg command in STATA version 10.1. The crosssectional analyses of free play interaction and ESCS were conducted using PRM or, in cases of significant overdispersion, the NBRM, utilizing the poisson or nbreg commands in STATA. Count models readily incorporate individual differences in the length of interaction via an 'exposure' variable. The duration of the free play interaction and of the ESCS, the total number of pointing trials administered in the ESCS, and the total number of 1-s gaze intervals and 5-s affect intervals coded per still face episode were included in the analysis as exposure variables. Age, site, and gender were included as potential covariates in all analyses. There were no sex differences in any of the outcome measures. 
Table 3 Proportion of intervals infants spent in each gaze and affect category across episodes of the Still Face Procedure

\begin{tabular}{|c|c|c|c|c|c|c|c|c|c|}
\hline & \multicolumn{3}{|c|}{$\operatorname{ASD}(n=9)$} & \multicolumn{3}{|c|}{ NoASD-sib $(n=46)$} & \multicolumn{3}{|l|}{$\mathrm{TD}(n=28)$} \\
\hline & Play & Still & Reunion & Play & Still & Reunion & Play & Still & Reunion \\
\hline \multicolumn{10}{|l|}{ Gaze } \\
\hline Mother's face & $48.3(26.1)$ & $26.9(11.9)$ & $44.2(27.1)$ & $40.5(26.7)$ & $24.6(25.2)$ & $35.6(24.3)$ & $39.8(21.4)$ & $20.7(17.9)$ & $35.1(22.6)$ \\
\hline Mother's body & $11.1(17.3)$ & $0.4(0.7)$ & $6.0(8.2)$ & $9.5(14.1)$ & $0.62(2.6)$ & $6.4(10.4)$ & $8.9(11.3)$ & $4.1(15.1)$ & $4.3(11.2)$ \\
\hline Away/avert & $39.0(27.3)$ & $63.0(7.2)$ & $30.2(18.3)$ & $44.2(26.4)$ & $62.2(26.9)$ & $50.0(27.1)$ & $48.1(23.9)$ & $64.5(22.1)$ & $56.6(26.3)$ \\
\hline Object & $0.17(0.5)$ & $8.3(13.3)$ & $16.2(31.8)$ & $2.9(7.1)$ & $11.8(19.6)$ & $4.8(9.8)$ & $1.8(6.8)$ & $9.4(16.3)$ & $1.3(4.0)$ \\
\hline Eyes closed & $1.5(3.9)$ & $0.4(0.7)$ & $1.1(2.6)$ & $1.6(4.6)$ & $0.75(3.4)$ & $1.6(7.2)$ & $1.1(3.9)$ & $1.2(4.2)$ & $1.6(6.9)$ \\
\hline \multicolumn{10}{|l|}{ Affect } \\
\hline Positive & $45.7(25.0)$ & $1.9(3.7)$ & $31.7(32.7)$ & $39.2(29.7)$ & $4.6(8.4)$ & $22.1(23.2)$ & $26.8(23.9)$ & $3.3(6.2)$ & $19.9(20.8)$ \\
\hline Neutral & $54.3(25.0)$ & $81.1(28.0)$ & $53.7(30.8)$ & $56.3(28.7)$ & $86.3(15.4)$ & $65.2(28.7)$ & $71.3(25.2)$ & $82.1(25.9)$ & $62.5(31.8)$ \\
\hline Negative & 0.00 & $17.1(29.0)$ & $14.7(25.6)$ & $3.6(10.2)$ & $8.9(14.3)$ & $12.3(24.8)$ & $1.4(6.4)$ & $14.5(25.2)$ & $17.2(32.8)$ \\
\hline
\end{tabular}

All data is presented in the format Mean (SD)

\section{Results}

\section{Still Face Procedure}

SFP analyses included 9 infants in the ASD group, 46 infants in the NoASD-sib group, and 28 infants in the TD group. Descriptive statistics for percent durations of infant gaze and affect categories are presented in Table 3. Instances of eyes closed, gaze to object, and gaze to mother's body were relatively infrequent; thus, our analysis focused on gaze to the mother's face and gaze aversion. Most infants spent the majority of the time in neutral affect; thus, analyses focused on positive and negative affect, as these captured more of the individual differences among infants. ${ }^{4}$

We conducted separate negative binomial regressions for each gaze and affect category of interest, with time and group main effects, time $\times$ group interactions, and age, site, and gender as potential covariates (see Table 4). Only statistically significant covariates are reported in the analyses below. The dependent variables included the number of 1-s intervals (gaze) and the number of 5-s intervals (affect) coded into the gaze and affect category of interest.

\section{Gaze Analyses}

The full NBRM model for gaze to the mother's face, with age as a covariate, was significant. The overall group by episode interaction was not significant, $\left(\chi_{(4)}^{2}=0.73\right.$, $p=.95)$; thus, we present the main effects analysis, with age retained as a covariate $\left(\chi_{(5)}^{2}=59.38, p<.001\right)$. There was a trend toward a significant group effect $\left(\chi_{(2)}^{2}=5.34\right.$, $p=.07)$, with infants in the ASD group demonstrating greater amounts of gaze to the mother's face across the

\footnotetext{
${ }^{4}$ Preliminary analyses indicated no group difference in neutral affect, both across the entire SFP as well as within individual episodes.
}

three still face episodes compared to the TD group (see Fig. 1). The main effect of episode was significant $\left(\chi_{(2)}^{2}=45.64, p<.001\right)$. Across the three groups, there was a significant decrease in gaze toward the mother's face from the play episode to the still face episode and a rebound of gaze to the mother's face to near-baseline levels in the reunion episode confirming the presence of still face and reunion effects.

The NBRM for gaze away from the mother was significant. However, the overall interaction of group and episode was not significant $\left(\chi_{(4)}^{2}=5.29, p=.26\right)$. Main effects analysis $\left(\chi_{(4)}^{2}=35.44, p<.001\right)$ indicated no significant effect of group on the amount of gaze aversion across the SFP $(z=2.39, p=.3)$. The main effect of episode was significant $\left(\chi_{(2)}^{2}=36.9, p<.001\right)$. Infants spent significantly more time with averted gaze during the still face compared to the play episode, and the amount of gaze aversion in the reunion rebounded to baseline levels, confirming the presence of the still face and reunion effects in this sample (see Fig. 1).

\section{Affect Analyses}

The full NBRM for positive affect, with site as a covariate, was significant. Because the group by episode interaction was not significant, $\left(\chi_{(4)}^{2}=3.98, p=.41\right)$, we present the main effects analysis, with site retained as a covariate $\left(\chi_{(5)}^{2}=125.48, p<.001\right)$. The main effect of group was not significant $\left(\chi_{(2)}^{2}=2.64, p=.27\right)$, indicating no group differences in infants' display of positive affect in the SFP. The main effect of episode was significant $\left(\chi_{(2)}^{2}=113.27\right.$, $p<.001)$. Infants spent significantly less time smiling in the still-face episode compared to the play episode, and their positive affect in the reunion episode remained below baseline levels demonstrating the carry-over effects of the still face (see Fig. 1). 
Table 4 Negative binomial regression on multiple still face outcomes

\begin{tabular}{|c|c|c|c|c|c|c|}
\hline Outcome & Covariate & IRR & SE & $z$-Score & $p$-value & $95 \% \mathrm{CI}$ \\
\hline \multirow[t]{5}{*}{ Gaze to face } & Still episode & 0.54 & 0.05 & -6.56 & $<.001$ & $0.45,0.65$ \\
\hline & Reunion episode & 0.92 & 0.07 & -1.02 & .31 & $0.78,1.08$ \\
\hline & ASD & 1.4 & 0.28 & 1.69 & .09 & $0.95,2.08$ \\
\hline & NoASD-sib & 0.9 & 0.12 & -0.78 & .44 & $0.70,1.17$ \\
\hline & Age & 0.75 & 0.09 & -2.26 & .02 & $0.59,0.96$ \\
\hline \multirow[t]{4}{*}{ Gaze avert/away } & Still episode & 1.5 & 0.11 & 5.45 & $<.001$ & $1.30,1.74$ \\
\hline & Reunion episode & 1.14 & 0.09 & 1.60 & .11 & $0.97,1.34$ \\
\hline & ASD & 0.82 & 0.11 & -1.46 & .14 & $0.63,1.07$ \\
\hline & NoASD-sib & 0.89 & 0.08 & -1.31 & .19 & $0.76,1.06$ \\
\hline \multirow[t]{5}{*}{ Positive affect } & Still episode & 0.11 & 0.02 & -10.47 & $<.001$ & $0.07,0.16$ \\
\hline & Reunion episode & 0.66 & 0.08 & -3.61 & $<.001$ & $0.53,0.83$ \\
\hline & ASD & 1.46 & 0.44 & 1.27 & .20 & $0.81,2.64$ \\
\hline & NoASD-sib & 1.33 & 0.26 & 1.45 & .15 & $0.90,1.97$ \\
\hline & Site & 1.89 & 0.41 & 2.94 & .003 & $1.24,2.89$ \\
\hline \multirow[t]{5}{*}{ Negative affect } & Still episode & 5.78 & 2.04 & 4.97 & $<.001$ & $2.89,11.56$ \\
\hline & Reunion episode & 5.59 & 2.07 & 4.63 & $<.001$ & $2.70,11.56$ \\
\hline & ASD & 0.93 & 0.68 & -0.1 & .92 & $0.22,3.92$ \\
\hline & NoASD-sib & 0.63 & 0.28 & -1.03 & .30 & $0.26,1.52$ \\
\hline & Site & 0.40 & 0.18 & -2.00 & .05 & $0.34,21.03$ \\
\hline
\end{tabular}

The NBRM predicting negative affect was significant. The overall interaction, however, was not significant, $\chi_{(4)}^{2}=3.88, p=.42$. Results of the main effect analysis with site as a covariate $\left(\chi_{(5)}^{2}=36.79, p<.001\right)$ indicated a non-significant group effect $\left(\chi_{(2)}^{2}=1.15, p=.56\right)$, and a significant effect of episode $\left(\chi_{(2)}^{2}=25.65, p<.001\right)$. As can be seen in Fig. 1, infants showed significantly more negative affect in the still face compared to the baseline play episode, with the amount of negative affect remaining elevated relative to baseline in the reunion episode.

\section{Mother-Infant Free Play Interaction}

The sample included 8 infants in the ASD group, 41 infants in the NoASD-sib group, and 35 infants in the TD group. Descriptive statistics of rates (per minute) and percent durations of individual infant behaviors are presented in Table 5. Since group differences were hypothesized specifically with regard to socially-directed behaviors, analyses focused on gaze to mother's face, and smiles and vocalizations co-occurring with gaze toward mother's face (social smile, social vocalization). ${ }^{5}$ The dependent variables in the PRM and NBRM analyses included the total

\footnotetext{
5 These variables were not mutually exclusive. Correlations between the rate of looks to the mother's face and the rate of social smiles $(r=.05)$ and social vocalizations $(r=-.12)$ were not significant $(p>.3)$. The correlation between social vocalization and social smile $(r=.26)$ was significant $(p=.01)$. Correlations among percent durations of these variables were all significant at $p<.001$ level, and ranged from .42 to .69 .
}

number of times the child produced the behavior of interest. There were no effects of age, site, and gender on rates and percent durations of infants' socio-communicative behaviors.

The PRM predicting gaze to the mother's face from group status was not significant $\left(\chi_{(2)}^{2}=0.16, p=.93\right)$, indicating no impact of our diagnostic outcome grouping on the frequency with which infants directed looks toward their mother's face. Similarly, NBRM analyses indicated no effect of group on rates of infant social smiles $\left(\chi_{(2)}^{2}=1.3, p=.52\right)$ and social vocalizations $\left(\chi_{(2)}^{2}=3.63\right.$, $p=.16$ ). Thus, the three groups did not differ in the frequency with which they directed looks, smiles, and vocalizations toward their mothers.

With regard to the duration of infants' socially-directed behaviors, one-way ANOVA analyses revealed no group differences in the proportion of time infants spent looking toward their caregiver's face, $F(2,81)=1.12, p=.3$. The variables representing percent durations of infant social smiles and social vocalizations were non-normally distributed with significant positive skew; hence, these variables were analyzed using nonparametric tests (Kruskal-Wallis). There were no group differences in the proportion of time infants smiled $\left(\chi_{(2)}^{2}=1.69, p=.43\right)$ and vocalized $\left(\chi_{(2)}^{2}=3.41, p=.18\right)$ while looking at their mothers' face.

\section{Nonverbal Communication}

The sample available for analyses consisted of 17 infants in the ASD group, 81 infants in the NoASD-sib group, and 60 
(a)

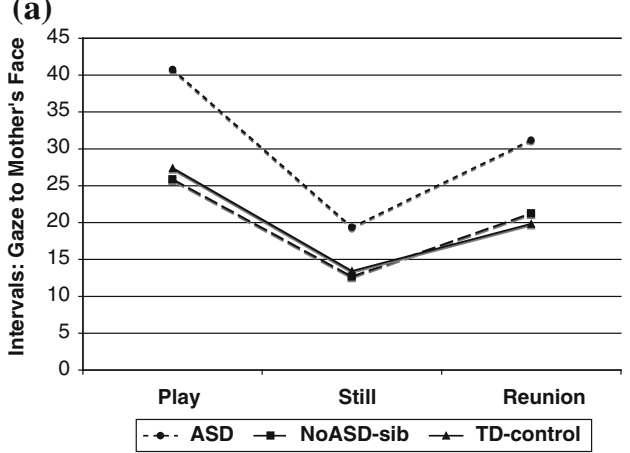

(c)

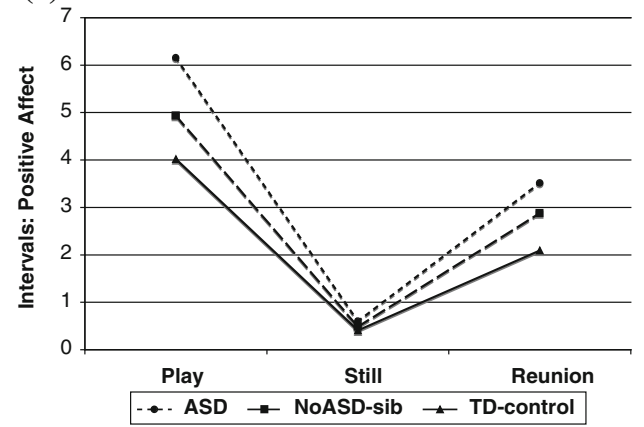

(b)

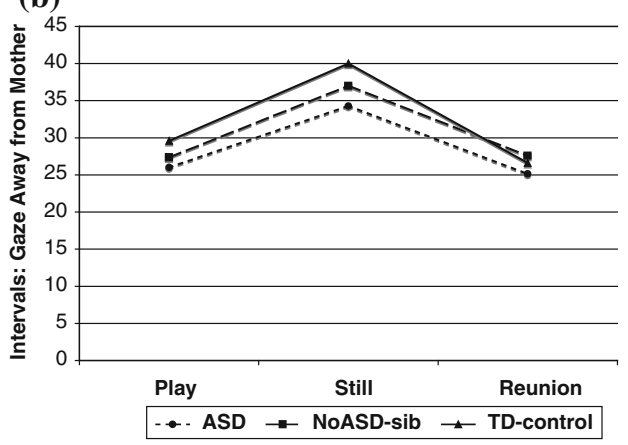

(d)

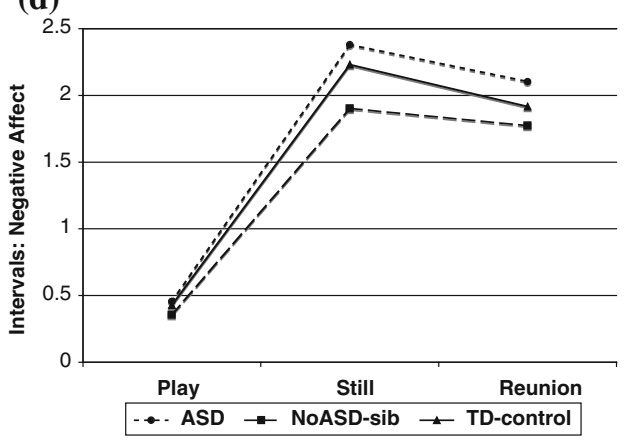

Fig. 1 Predicted number of 1-s (gaze) and 5-s (affect) intervals spent in each gaze and affect category of interest in the still face procedure. a Gaze to mother's face; b Gaze away from the mother; c Positive affect; $\mathbf{d}$ Negative affect

Table 5 Rates and durations of infant behaviors in the face-to-face interaction

\begin{tabular}{|c|c|c|c|c|c|c|}
\hline & \multicolumn{2}{|c|}{$\operatorname{ASD}(n=8)$} & \multicolumn{2}{|c|}{ NoASD-sib $(n=41)$} & \multicolumn{2}{|c|}{$\mathrm{TD}(n=35)$} \\
\hline & Rate & $\%$ Duration & Rate & $\%$ Duration & Rate & $\%$ Duration \\
\hline Gaze face & $6.6(3.0)$ & $56.4(27.8)$ & $6.5(2.4)$ & $43.0(22.0)$ & $6.6(2.5)$ & $43.8(24.3)$ \\
\hline Smile & $4.4(2.3)$ & $39.1(23.9)$ & $4.2(2.0)$ & $29.9(21.8)$ & $3.8(2.5)$ & $29.4(25.5)$ \\
\hline Social smile & $3.6(2.6)$ & $33.4(26.3)$ & $3.0(1.9)$ & $21.3(17.8)$ & $2.8(2.0)$ & $21.2(20.1)$ \\
\hline Vocalization & $2.0(2.7)$ & $3.2(5.6)$ & $3.7(3.8)$ & $7.1(9.9)$ & $4.1(4.5)$ & $8.9(12.5)$ \\
\hline Social voc. & $0.6(1.0)$ & $0.7(1.2)$ & $2.3(2.8)$ & $4.4(5.8)$ & $2.3(3.1)$ & $5.0(8.5)$ \\
\hline
\end{tabular}

Rates (per minute) and percent durations are in the form Mean (SD)

infants in the TD group. Descriptive statistics regarding rates of joint attention and requesting behaviors can be found in Table 6. All variables showed evidence of overdispersion; hence, we conducted separate Negative Binomial regressions for each variable of interest, with age, site, and gender as potential covariates (see Table 7). Only statistically significant covariates are reported in the analyses below. The length of the ESCS administration and number of RJA point trials administered were included in the analyses as exposure variables.

\section{Group Differences in Initiating Joint Attention}

The NBRM predicting IJA_low from outcome group status and site was statistically significant $\left(\chi_{(3)}^{2}=10.78\right.$,
Table 6 Rates of joint attention and requesting behaviors at 12 months

\begin{tabular}{llll}
\hline & ASD $(n=17)$ & NoASD-sib $(n=81)$ & TD $(n=60)$ \\
\hline IJA_low & $1.08(0.59)$ & $1.16(0.55)$ & $1.09(0.58)$ \\
IJA_high & $0.04(0.12)$ & $0.11(0.17)$ & $0.13(0.15)$ \\
IBR_low & $0.39(0.21)$ & $0.61(0.36)$ & $0.64(0.38)$ \\
IBR_high & $0.13(0.16)$ & $0.44(0.33)$ & $0.41(0.39)$ \\
RJA_dist (\%) & $11.8(18.4)$ & $34.2(32.0)$ & $28.8(29.4)$ \\
RJA_prox (\%) & $57.1(33.4)$ & $59.4(25.1)$ & $62.9(27.3)$
\end{tabular}

IJA and IBR variables are expressed as a rate per minute; RJA variables are expressed as a percentage of correct responses. All variables are in the form Mean (SD) 
Table 7 Negative binomial regression on joint attention and behavior regulation variables

\begin{tabular}{llllccl}
\hline Outcome & Covariate & IRR & SE & $z$-score & $p$-value & $95 \%$ CI \\
\hline IJA_low & ASD & 1.01 & 0.14 & 0.10 & .92 & $0.77,1.3$ \\
& NoASD-sib & 1.05 & 0.09 & 0.55 & .58 & $0.87,1.24$ \\
& Site & 0.73 & 0.07 & -3.27 & .001 & $0.60,0.88$ \\
IJA_high & ASD & 0.31 & 0.15 & -2.36 & .02 & $0.12,0.82$ \\
& NoASD-sib & 0.88 & 0.24 & -0.46 & .65 & $0.52,1.5$ \\
IBR_low & ASD & 0.62 & 0.1 & -2.91 & .004 & $0.45,0.86$ \\
& NoASD-sib & 0.96 & 0.09 & -0.46 & .65 & $0.79,1.16$ \\
IBR_high & ASD & 0.3 & 0.09 & -3.93 & $<.001$ & $0.17,0.56$ \\
& NoASD-sib & 1.09 & 0.18 & 0.60 & .6 & $0.79,1.51$ \\
RJA_dist & ASD & 0.43 & 0.16 & 2.32 & .02 & $0.21,0.88$ \\
& NoASD-sib & 1.15 & 0.20 & 0.80 & .43 & $0.82,1.6$ \\
& Age & 1.65 & 0.36 & 2.29 & .02 & $1.07,2.52$ \\
& Site & 2.12 & 0.37 & 4.29 & $<.001$ & $1.51,3.0$ \\
RJA_prox & ASD & 0.91 & 0.11 & -0.74 & .46 & $0.71,1.17$ \\
& NoASD-sib & 0.95 & 0.07 & -0.68 & .50 & $0.82,1.1$
\end{tabular}

$\overline{I R R}$ incidence rate ratio, $S E$ standard error of IRR, $C I$ confidence interval. Reference category is the TD group

$p=.01$ ). Controlling for site, there were no differences between the three groups in rates of low-level joint attention bids. The predicted rates for low-level joint attention bids were 1.08 for the ASD group, 1.14 for the NoASD-sib group, and 1.08 for the TD group. Thus, on average, infants across the three groups produced about one low-level joint attention bid per minute.

The NBRM predicting IJA_high from group was marginally significant $\left(\chi_{(2)}^{2}=5.1, \bar{p}=.07\right)$. Infants in the ASD group had lower expected rates of joint attention points and shows relative to infants in the TD group $(z=-2.36$, $p=.02)$ and infants in the NoASD-sib group $(z=-2.15$, $p=.03$ ). There were no differences between the NoASDsib group and the TD group $(z=-0.46, p=.65)$. The predicted rate of high-level joint attention bids for the NoASD-sib group was .11 and for the TD group was 0.13 , which amounts to about 1 bid per 9 min. In contrast, the predicted rate for the ASD group was 0.04 , or about 1 bid per $25 \mathrm{~min}$.

\section{Group Differences in Initiating Behavior Regulation}

The NBRM predicting IBR_low from group status was statically significant $\left(\chi_{(2)}^{2}=8.2, p=.02\right)$. The ASD group had lower expected rates of IBR_low than TD group $(z=$ $-2.91, p=.004)$ and the NoASD-sib group $(z=-2.7$, $p=.007)$. There were no differences between the NoASDsibs and the TD group $(z=-0.46, p=.65)$. The predicted rates for the NoASD-sibs and TD infants were 0.61 and 0.63 , respectively (about 6 bids per $10 \mathrm{~min}$ ), whereas the predicted rate for the ASD group was 0.4 , or about 4 bids per $10 \mathrm{~min}$.

Finally, the NBRM predicting IBR_high from group status was also statically significant $\left(\chi_{(2)}^{2}=15.8\right.$, $p<.001)$. The ASD infants had lower expected rates of IBR_high than infants in the TD group $(z=-3.93$, $p<.001)$ and the NoASD-sib group $(z=-4.33$, $p<.001)$. There were no differences between the NoASDsibs and infants in the TD group $(z=0.53, p=.6)$. The predicted rate of high level requesting bids for infants in the NoASD-sib and TD groups was 0.4 , or 4 bids per $10 \mathrm{~min}$, whereas the predicted rate for the ASD group was 0.12 (1.2 bids per $10 \mathrm{~min}$ ).

\section{Group Differences in Responding to Joint Attention}

Group differences in infants' ability to follow the examiner's distal points to posters on the wall (RJA_dist) and proximal points to pictures in a book (RJA_prox) were analyzed using NBRM, with the number of points administered as the exposure variable. The model predicting rate of RJA_dist from group, with age and site as covariates, was significant, $\left(\chi_{(4)}^{2}=27.12, p<.001\right)$. Infants in the ASD group responded to significantly fewer of the examiner's distal points than NoASD-sibs $(z=2.32, p=.02)$ and infants in the TD group $(z=2.71, p=.007)$. There were no significant differences between the NoASD-sibs and TD infants $(z=.8, p=.43)$. There were no group differences in infants' capacity to follow the examiner's points to pictures in a book, $\chi_{(2)}^{2}=0.75, p=.69$.

\section{Discussion}

Across analyses reported herein, we replicated recent findings indicating little evidence that infants who are diagnosed with an ASD at 36 months of age can be differentiated from non-diagnosed autism siblings and lowrisk controls at 6 months of age on the basis of early disturbance in socially directed behaviors or in sensitivity to a disruption in contingent interaction with the caregiver. However, by 12 months, these infants exhibited delays in nonverbal communication that cut across joint attention and requesting domains. In contrast, in the group of siblings of children with autism who did not go on to meet diagnostic criteria for an ASD, we found no evidence of early deficits in social interaction at 6 months or in nonverbal communication at 12 months, which we expected to index the presence of the broader autism phenotype.

Contrary to our hypotheses, infants who were diagnosed with an ASD at 36 months were just as likely to direct gaze, smiles, and vocalizations toward their caregiver during a brief free play interaction at 6 months as low-risk 
controls. This finding, which held for both duration and frequency measures of the behaviors, was surprising given the well documented deficits in social gaze and social smiling, difficulties perceiving and interpreting the social cues of others, and deficits in emotional responsiveness among preschool age children with autism. Perhaps a more microanalytic analysis of the timing of infant behaviors in relation to the caregiver over a longer period of observation could reveal subtle qualitative differences in early social engagement of infants with autism, such as the lower synchrony in infant-led interactions among non-affected siblings previously documented by Yirmiya et al. (2006). Nonetheless, at present we suggest that global measures of the frequency and amount of time infants spend looking at their caregivers and coordinating their gaze with other communicative behaviors such as vocalizations and smiles, do not reveal evidence of autism-specific deficits at 6 months of age.

Infants subsequently diagnosed with an ASD were also equally sensitive to a disruption of contingent social interaction by their caregiver in the SFP, as evidenced by a decrease in smiling and gaze toward the mother's face and increased gaze aversion and negative affect when she withdrew from interaction. The caregiver's sudden withdrawal from interaction is an extremely powerful social cue for young infants (Adamson and Frick 2003), and thus the still face effect may in fact be too robust to capture early autism-specific deficits. Indeed, the sole group difference in the SFP was a statistical trend toward a greater amount of overall time spent looking toward the caregiver's face across the three episodes of the SFP by infants in the ASD group compared to infants in the other two groups. While this result echoes the Ibanez et al. (2008) finding of lower frequency of gaze shifts and longer mean durations of gaze at the mother's face among infants at-risk for ASD, it is important to note that a similar result was not observed in the same group of infants in the free play interaction, which included more detailed coding of the onsets and offsets of gaze shifts toward and away from the mother. Thus, the increased attention to the caregiver's face documented in the SFP was not a stable characteristic of infants subsequently diagnosed with ASD in the present study.

In sum, our results indicate that previous findings of reduced social orienting (Osterling and Dawson 1994; Osterling et al. 2002) and atypicalities in eye contact and social smiling (Zwaigenbaum et al. 2005) among 12-month-old infants subsequently diagnosed with autism cannot be extended downward to 6 months. Although the small number of affected infants reduced our statistical power to detect group differences at 6 months, our findings nonetheless substantiate the results from other longitudinal studies of infant siblings indicating no clear behavioral markers for ASD at 6 months (Landa et al. 2007; Ozonoff et al. 2010; Zwaigenbaum et al. 2005). Despite recognition that autism is a neurodevelopmental disorder, and evidence that the underlying brain abnormality may be present from birth (Bauman and Kemper 2005; Nelson et al. 2001; Rodier et al. 1996), the limited and largely undifferentiated behavioral repertoires of very young infants may not allow us to distinguish clinically meaningful behavioral signs of autism in infancy.

Measures of high level initiating joint attention, responding to joint attention, and requesting behaviors were sensitive to early manifestations of autism at 12 months of age. As predicted, infants subsequently diagnosed with an ASD were less likely to direct the examiner's attention to toys of interest via pointing and showing gestures and to respond to her attention-sharing bids than infants in the other two groups. These findings support current practices in autism screening, which incorporate a failure to point to objects, to hold objects up for others to see, and to respond to the pointing gestures of others as red flags for ASD in the first two years of life (Bryson et al. 2008; Lord et al. 2000; Robins et al. 2001; Stone et al. 2000). A lack of group differences in low-level joint attention behaviors, such as alternating gaze between an active toy and the examiner, was surprising. The highly structured setting of the ESCS may have proved optimal in eliciting joint attention looks from the infants, as researchers have noted that children with autism perform considerably better in situations where the number of stimuli is limited and presented in a routine, predictable manner (Dawson et al. 2000). An analysis of spontaneous joint attention bids in unstructured play settings, where the child is free to move about and there are multiple objects competing for his attention, is warranted prior to concluding that low-level joint attention behaviors are not disrupted within the first year of life among infants who go on to be diagnosed with autism.

Contrary to evidence from studies with preschool age children, which indicate deficits in joint attention but not requesting behaviors differentiate children with autism from neurotypical and developmentally delayed children (Sigman and Ruskin 1999), infants in the ASD group exhibited lower rates of both low and high level requesting behaviors relative to unaffected siblings and comparison infants. These findings complement those of Landa et al. (2007) who found that siblings who received a clinical judgment of ASD by 36 months could be distinguished from typically developing comparison infants and unaffected siblings at 14 months on the basis of less frequent initiation of both joint attention and requesting bids. Thus, what appears to characterize risk for autism at 12 months is not a specific failure to use gaze, affect, and gesture to share attention for purely social purposes, but rather a more general communicative deficit in using such behaviors to 
actively engage with others around both attention sharing and requesting contexts.

The question of diagnostic predictiveness of specific measures of joint attention and requesting requires further empirical attention. As is expected at this early age (Bakeman and Adamson 1984; Butterworth and Jarrett 1991), the low rate of responding on certain measures and large standard deviations around group means on others indicated large within-group variability in skill across both affected and unaffected infants. The desire to push the search for early markers for autism, and indeed the diagnosis itself, earlier in infancy will inevitably run up against the normative variability in the development of early social-communication skills. The fact that deficits in joint attention and requesting on a group level were identified in the current study at an age when these behaviors are still emerging is quite exciting, and provides new hope for efforts to identify appropriate treatment targets for infants and toddlers showing signs of autism (Kasari 2002).

Infants' capacity to enter into early communicative interactions allows them to learn from and through others about the environment. Early joint attention behaviors play a self-organizing function, whereby the child's tendency to initiate social attention coordination with others assures social information input and experience presumed crucial to promoting normative behavioral and neural development (Bernier et al. 2006; Klin et al. 2005; Mundy and Neal 2001). In particular, episodes of object-mediated joint engagement are deemed central to infants' developing understanding of others' goals and intentions, and to the emergence of language (Baldwin 1995; Tomasello 1995; Tomasello et al. 2005). Viewed through this lens, the deficits in initiating joint attention and requesting identified in the present study suggest that 12-month-old infants who go on to be diagnosed with ASD may be far less likely to create critical social experiences for themselves. The implications of such early deficits for the subsequent development are underscored by evidence that individual differences in the initiation of joint attention and gestures in general predict language (Charman et al. 2003; Mundy et al. 1990; Sigman and Ruskin 1999) and theory of mind task performance (Charman et al. 2000) among young children with autism, and prosocial behavior and play with peers among adolescents with autism (Sigman and Ruskin 1999; Travis et al. 2001).

Turning to our NoASD siblings, we found no evidence that domains that tap early manifestations of autism at 12 months represent areas of impairment for this group as a whole. Although at first glance our results contradict previously published reports of nonverbal communication delays in non-autistic siblings of children with autism, upon closer examination, the deficits reported in those studies are subtle and inconsistent. Toth et al. (2007) found evidence of lower overall rate of communicating and use of distal gestures but no differences in the frequency of specific behaviors such as gaze shifts, gaze/point following, and requesting and joint attention gestures from 18 to 25 months of age. Cassel et al. (2007) and Yirmiya et al. (2006) reported lower rates of high level requesting behaviors at 12 and 14 months, respectively, but these studies yielded conflicting findings with regard to IJA and RJA impairments at 14-15 months. Finally, findings by Landa et al. (2007) suggest that delays in RJA at 14 months may only be evident in those siblings who subsequently exhibit language delays or social impairments. Beyond methodological considerations, these results suggest there is little consensus regarding a specific profile of deficits in this group.

It is important to note that our analyses focused on investigating whether non-diagnosed siblings as a group experience delays in social skills and nonverbal communication. Although this is a common approach in the infant sibling literature (e.g., Cassel et al. 2007; Yirmiya et al. 2006; Toth et al. 2007), evidence from family studies suggests that only a subset of older siblings and family members may be affected by the BAP (Bailey et al. 1998; Bolton et al. 1994). The practice of exploring features of the BAP by considering all non-diagnosed siblings as potentially demonstrating deficits may in fact obscure distinct subgroups of affected siblings, and place undue emphasis on characterizing this group of children as a whole as being atypical. Another approach has been to create a BAP subgroup by identifying siblings who show language and/or social delays at outcome, and then to examine whether these infants exhibit signs of atypical development in infancy (e.g., Landa et al. 2007; Sullivan et al. 2007). However, to the extent that the question of delineating the BAP, both in terms of the developmental domains that are affected and in terms of the age at which it first emerges, is an empirical one, a priori defining the BAP based on selected outcome measures may also not represent the best approach.

Future research could rely on statistical clustering techniques to identify subgroups of non-autistic siblings that show deficits across multiple domains, including language and nonverbal communication, cognition, and social skills. Ultimately, any evidence of an early BAP involving social and communication deficits in infancy will need to be confirmed through longitudinal follow-up to investigate whether early deficits persist, and whether they have measurable consequences for subsequent social and emotional functioning.

Several limitations of the study bear acknowledgement. The composition of the sample varied across the analyses reported herein. Moreover, not all infants in the present sample were seen through the 36-month assessment visit. 
However, it must be noted that all of the infants in the ASD-group had been seen through 36 months of age, and of those infants in the NoASD-sib group who had been seen through the 24-month visit only, none exhibited any behaviors indicative of autism at 24 months based on clinician judgment. The final limitation concerns the small sample size in the affected sibling group for the 6-month analyses, though it should be noted that the means and standard deviations for rates and durations of communicative behaviors in the affected group are nearly identical as those in the other two groups. Nonetheless, the findings of the present study should be replicated with a larger sample of affected infants.

Acknowledgments This research was supported by NIMH/NIH Studies to Advance Autism Research and Treatment (STAART) program grant number U54-MH-068172 awarded to M. Sigman, P.I, grant number R01 MH068398 from the National Institute of Mental Health awarded to S. Ozonoff, P.I., and grants from the National Association for Autism Research (NAAR), from Cure Autism Now (CAN), and from the Medical Investigation of Neurodevelopmental Disorders Institute (M.I.N.D) awarded to S. J. Rogers. We extend a special thank you to Lily Altstein for her assistance with the statistical analyses, and to all the families that have kindly and generously given so much of their time to participate in this study.

Open Access This article is distributed under the terms of the Creative Commons Attribution Noncommercial License which permits any noncommercial use, distribution, and reproduction in any medium, provided the original author(s) and source are credited.

\section{References}

Adamson, L. B., \& Frick, J. E. (2003). The still face: A history of a shared experimental paradigm. Infancy, 4, 451-473.

APA. (1994). Diagnostic and statistical manual of mental disorders (4th ed.). Washington, DC: Author.

Bailey, A., Palferman, S., Heavey, L., \& Le Couteur, A. (1998). Autism: The phenotype in relatives. Journal of Autism and Developmental Disorders, 28(5), 369-392.

Bakeman, R., \& Adamson, L. B. (1984). Coordinating attention to people and objects in mother infant and peer-infant interaction. Child Development, 55(4), 1278-1289.

Baldwin, D. A. (1995). Understanding the link between joint attention and language. In C. Moore \& P. J. Dunham (Eds.), Joint attention: Its origins and role in development (pp. 131-158). Hillsdale, NJ: Lawrence Erlbaum.

Baranek, G. T. (1999). Autism during infancy: A retrospective video analysis of sensory-motor and social behaviors at 9-12 months of age. Journal of Autism and Developmental Disorders, 29(3), 213-224.

Bauman, M. L., \& Kemper, T. L. (2005). Neuroanatomic observations of the brain in autism: A review and future directions. International Journal of Developmental Neuroscience, 23(2-3), 183-187.

Bernier, R., Webb, S. J., \& Dawson, G. (2006). Understanding impairments in social engagement in autism. In P. J. Marshall \& N. A. Fox (Eds.), The development of social engagement:
Neurobiological perspectives (pp. 304-330). New York: Oxford University Press.

Bolton, P., Macdonald, H., Pickles, A., \& Rios, P. (1994). A casecontrol family history study of autism. Journal of Child Psychology and Psychiatry, 35(5), 877-900.

Bruner, J., \& Sherwood, V. (1983). Thought, language and interaction in infancy. In J. D. Call, E. Galenson, \& R. L. Tyson (Eds.), Frontiers of infant psychiatry. New York: Basic Books.

Bryson, S., Zwaigenbaum, L., McDermott, C., Rombough, V., \& Brian, J. (2008). The Autism Observation Scale for Infants: Scale development and reliability data. Journal of Autism and Developmental Disorders, 38, 731-738.

Butterworth, G., \& Jarrett, N. (1991). What minds have in common is space: Spatial mechanisms serving joint visual attention in infancy. British Journal of Developmental Psychology, 9, 55-72.

Carpenter, M., Nagell, K., \& Tomasello, M. (1998). Social cognition, joint attention, and communicative competence from 9 to 15 months of age. Monographs of the Society for Research in Child Development, 63 (4, Serial No. 255).

Cassel, T. D., Messinger, D., Ibanez, L. V., Haltigan, J. D., Acosta, S. I., \& Buchman, A. C. (2007). Early social and emotional communication in infant siblings of children with autism spectrum disorders: An examination of the broad phenotype. Journal of Autism and Developmental Disorders, 37(1), 122-132.

Charman, T., Baron-Cohen, S., Swettenham, J., Baird, G., Cox, A., \& Drew, A. (2000). Testing joint attention, imitation, and play as infancy precursors to language and theory of mind. Cognitive Development, 15(4), 481-498.

Charman, T., Baron-Cohen, S., Swettenham, J., Baird, G., Drew, A., \& Cox, A. (2003). Predicting language outcome in infants with autism and pervasive developmental disorder. International Journal of Language \& Communication Disorders, 38(3), 265-285.

Charman, T., Swettenham, J., Baron-Cohen, S., Cox, A., Baird, G., \& Drew, A. (1997). Infants with autism: An investigation of empathy, pretend play, joint attention, and imitation. Developmental Psychology, 33(5), 781-789.

Cohn, J., \& Tronick, E. (1988). Mother-infant face-to-face interaction: Influence is bidirectional and unrelated to periodic cycles in either partner's behavior. Developmental Psychology, 24(3), 386-392.

Dawson, G., Hill, D., Spencer, A., Galpert, L., \& Watson, L. (1990). Affective exchanges between young autistic children and their mothers. Journal of Abnormal Child Psychology, 18(3), $335-345$.

Dawson, G., Osterling, J., Meltzoff, A. N., \& Kuhl, P. (2000). Case study of the development of an infant with autism from birth to two years of age. Journal of Applied Developmental Psychology, 21(3), 299-313.

Dawson, G., Toth, K., Abbott, R., Osterling, J., Munson, J., Estes, A., et al. (2004). Early social attention impairments in autism: Social orienting, joint attention, and attention to distress. Developmental Psychology, 40, 271-283.

Feldman, R. (2003). Infant-mother and infant-father synchrony: The co-regulation of positive arousal. Infant Mental Health Journal, $24(1), 1-23$.

Fombonne, E. (2003). Epidemiological surveys of autism and other pervasive developmental disorders: An update. Journal of Autism and Developmental Disorders, 33(4), 365-382.

Hains, S. M. J., \& Muir, D. W. (1996). Effects of stimulus contingency in infant-adult interactions. Infant Behavior \& Development, 19(1), 49-61.

Ibanez, L. V., Messinger, D. S., Newell, L., Lambert, B., \& Sheskin, M. (2008). Visual disengagement in the infant siblings of 
children with an autism spectrum disorder (ASD). Autism, 12(5), 473-485.

Jaffe, J., Beebe, B., Stanley, F., Crown, C. L., \& Jasnow, M. D. (2001). Rhythms of dialogue in infancy: Coordinated timing in development. Monographs of the Society for Research in Child Development, 66(2), 131.

Joseph, R. M., \& Tager-Flusberg, H. (1997). An investigation of attention and affect in children with autism and Down syndrome. Journal of Autism and Developmental Disorders, 27(4), 385-396.

Kasari, C. (2002). Assessing change in early intervention programs for children with autism. Journal of Autism and Developmental Disorders, 32(5), 447-461.

Kasari, C., Sigman, M., Mundy, P., \& Yirmiya, N. (1990). Affective sharing in the context of joint attention interactions of normal, autistic, and mentally retarded children. Journal of Autism and Developmental Disorders, 20(1), 87-100.

Klin, A., Jones, W., Schultz, R., \& Volkmar, F. (2005). The enactive mind-from actions to cognition: Lessons from autism. In F. Volkmar, P. Rhea, A. Klin, \& D. Cohen (Eds.), Handbook of autism and pervasive developmental disorders, vol. 1: Diagnosis, development, neurobiology, and behavior (pp. 682-703). NJ: Hoboken.

Landa, R., Holman, K. C., \& Garrett-Mayer, E. (2007). Social and communication development in toddlers with early and later diagnosis of autism spectrum disorders. Archives of General Psychiatry, 64(7), 853-864.

Long, J. S., \& Freese, J. (2006). Regression models for categorical outcomes using stata (2nd ed.). College Station, TX: Stata Press.

Lord, C., Risi, S., Lambrecht, L., Cook, E. H., Leventhal, B. L., DiLavore, P. C., et al. (2000). The autism diagnostic observation schedule-generic: A standard measure of social and communication deficits associated with the spectrum of autism. Journal of Autism and Developmental Disorders, 30(3), 205-223.

Lord, C., Rutter, M., \& Le Couteur, A. (1994). Autism diagnostic interview-revised: A revised version of a diagnostic interview for caregivers of individuals with possible pervasive developmental disorders. Journal of Autism and Developmental Disorders, 24(5), 659-685.

Merin, N., Young, G. S., Ozonoff, S., \& Rogers, S. J. (2007). Visual fixation patterns during reciprocal social interaction distinguish a subgroup of 6-month-old infants at-risk for autism from comparison infants. Journal of Autism and Developmental Disorders, 37, 108-121.

Messinger, D. S., Fogel, A., \& Dickson, K. L. (1999). What's in a smile? Developmental Psychology, 35(3), 701-708.

Mitchell, S., Brian, J., Zwaigenbaum, L., Roberts, W., Szatmari, P., Smith, I., et al. (2006). Early language and communication development of infants later diagnosed with autism spectrum disorder. Journal of Developmental and Behavioral Pediatrics, 27(Suppl 2), S69-S78.

Mullen, E. M. (1995). Mullen Scales of Early Learning. Circle Pines, $\mathrm{MN}$ : American Guidance Service.

Mundy, P., Delgado, C., Block, J., Venezia, M., Hogan, A., \& Siebert, J. (2003). A manual for the abridged early social communication scales (ESCS). Unpublished.

Mundy, P., \& Neal, A. R. (2001). Neural plasticity, joint attention, and a transactional social-orienting model of autism. In L. M. Glidden (Ed.), International review of research in mental retardation: Autism (pp. 139-168). San Diego: Academic Press.

Mundy, P., Sigman, M., \& Kasari, C. (1990). A longitudinal study of joint attention and language development in autistic children. Journal of Autism and Developmental Disorders, 20(1), $115-128$.

Mundy, P., Sigman, M. D., Ungerer, J., \& Sherman, T. (1986). Defining the social deficits of autism: The contribution of non- verbal communication measures. Journal of Child Psychology and Psychiatry, 27(5), 657-669.

Nelson, K. B., Grether, J. K., Croen, L. A., Dambrosia, J. M., Dickens, B. F., Jelliffe, L. L., et al. (2001). Neuropeptides and neurotrophins in neonatal blood of children with autism or mental retardation. Annals of Neurology, 49, 597-606.

Osterling, J., \& Dawson, G. (1994). Early recognition of children with autism: A study of first birthday home videotapes. Journal of Autism and Developmental Disorders, 24(3), 247-257.

Osterling, J., Dawson, G., \& Munson, J. A. (2002). Early recognition of 1-year-old infants with autism spectrum disorder versus mental retardation. Development and Psychopathology, 14(2), 239-251.

Ozonoff, S., Iosif, A., Baguio, F., Cook, I. C., Hill, M. M., Hutman, T., et al. (2010). A prospective study of the emergence of early behavioral signs of autism. Journal of the American Academy of Child and Adolescent Psychiatry, 49, 258-268.

Pickles, A., Starr, E., Kazak, S., Bolton, P., Papanikolaou, K., Bailey, A., et al. (2000). Variable expression of the autism broader phenotype: Findings from extended pedigrees. Journal of Child Psychology and Psychiatry, 41(4), 491-502.

Ritvo, E. R., Jorde, L. B., Mason-Brothers, A., Freeman, B. J., Pingree, C., Jones, M. B., et al. (1989). The UCLA-University of Utah epidemiologic survey of autism: Recurrence risk estimates and genetic counseling. American Journal of Psychiatry, 146, $1032-1036$.

Robins, D. L., Fein, D., Barton, M., \& Green, J. (2001). The modified checklist for autism in toddlers: An initial study investigating the early detection of autism and pervasive developmental disorders. Journal of Autism and Developmental Disorders, 31(2), 131-144.

Rodier, P. M., Ingram, J. L., Tisdale, B., Nelson, S., \& Romano, J. (1996). Embryological origin for autism: Developmental anomalies of the cranial nerve motor nuclei. Journal of Comparative Neurology, 370(2), 247-261.

Rutter, M., Bailey, A., \& Lord, C. (2003). Social communication questionnaire. Los Angeles, CA: Western Psychological Services.

Shrout, P. E., \& Fleiss, J. L. (1979). Intraclass correlation: Uses in assessing rater reliability. Psychological Bulletin, 86, 420-428.

Sigman, M., \& Ruskin, E. (1999). Continuity and change in the social competence of children with autism, Down syndrome, and developmental delays. Monographs of the Society for Research in Child Development, 64(1), v-114.

Stone, W. L., Coonrod, E. E., \& Ousley, O. Y. (2000). Brief report: Screening tool for autism in two-year-olds (STAT): Development and preliminary data. Journal of Autism and Developmental Disorders, 30(607-612), 691-701.

Sullivan, M., Finelli, J., Marvin, A., Garrett-Mayer, E., Bauman, M., \& Landa, R. (2007). Response to joint attention in toddlers at risk for autism spectrum disorder: A prospective study. Journal of Autism and Developmental Disorders, 37(1), 37-48.

Tomasello, M. (1995). Joint attention as social cognition. In C. Moore \& P. J. Dunham (Eds.), Joint attention: Its origins and role in development (pp. 103-130). Hillsdale, NJ: Lawrence Erlbaum Associates.

Tomasello, M., Carpenter, M., Call, J., Behne, T., \& Moll, H. (2005). Understanding and sharing intentions: The origins of cultural cognition. Behavioral and Brain Sciences, 28(5), 675-735.

Toth, K., Dawson, G., Meltzoff, A. N., Greenson, J., \& Fein, D. (2007). Early social, imitation, play, and language abilities of young non-autistic siblings of children with autism. Journal of Autism and Developmental Disorders, 37(1), 145-157.

Trad, P. V., Bernstein, D., Shapiro, R., \& Hertzig, M. (1993). Assessing the relationship between affective responsivity and social interaction in children with pervasive developmental 
disorder. Journal of Autism and Developmental Disorders, 23(2), 361-377.

Travis, L., Sigman, M., \& Ruskin, E. (2001). Links between social understanding and social behavior in verbally able children with autism. Journal of Autism and Developmental Disorders, 31(2), 119-130.

Tronick, E., Als, H., Adamson, L. B., Wise, S., \& Brazelton, T. B. (1978). The infant's response to entrapment between contradictory messages in face-to-face interaction. Journal of Child Psychiatry, 17, 1-13.

Tronick, E., \& Cohn, J. F. (1989). Infant-mother face-to-face interaction: Age and gender differences in coordination and the occurrence of miscoordination. Child Development, 60(1), 85-92.

Weinberg, K., \& Tronick, E. (1994). Beyond the face: An empirical study of infant affective configurations of facial, vocal, gestural, and regulatory behaviors. Child Development, 65(5), 1503-1515.
Yale, M. E., Messinger, D. S., Cobo-Lewis, A. B., \& Delgado, C. F. (2003). The temporal coordination of early infant communication. Developmental Psychology, 39(5), 815-824.

Yirmiya, N., Gamliel, I., Pilowsky, T., Feldman, R., Baron-Cohen, S., \& Sigman, M. (2006). The development of siblings of children with autism at 4 and 14 months: Social engagement, communication, and cognition. Journal of Child Psychology and Psychiatry, 47(5), 511-523.

Young, G. S., Merin, N., Rogers, S. J., \& Ozonoff, S. (2009). Gaze behavior and affect at 6 months: Predicting clinical outcomes and language development in typically developing infants and infants at risk for autism. Developmental Science, 12, 798-814.

Zwaigenbaum, L., Bryson, S., Rogers, T., Roberts, W., Brian, J., \& Szatmari, P. (2005). Behavioral manifestations of autism in the first year of life. International Journal of Developmental Neuroscience, 23(2-3), 143-152. 